Tecno Lógicas

ISSN 0123-7799

Vol. 17, No. 32, pp. 33-46

Enero-junio de 2014
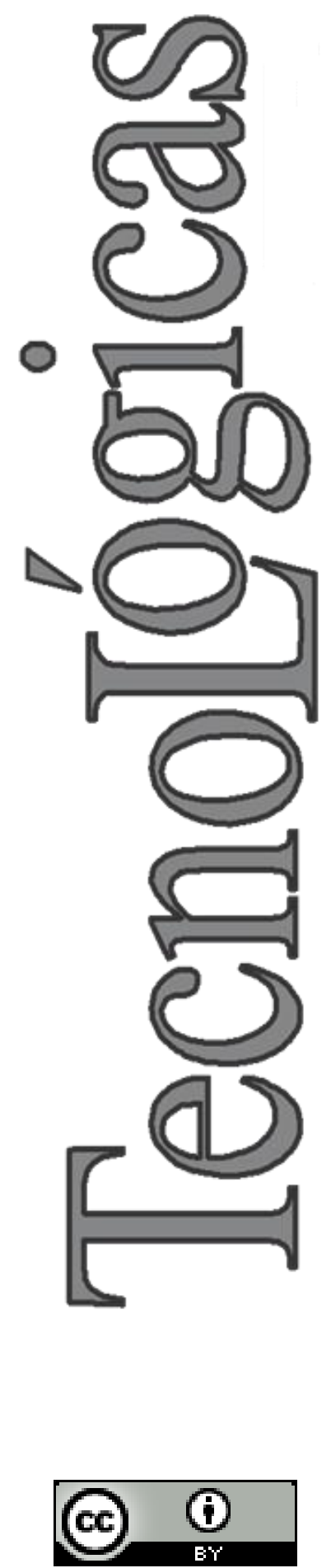

\section{Control global del péndulo con rueda de reacción mediante regulación de energía y linealización extendida de las variables de estado}

\section{Global control of reaction wheel pendulum through energy regulation and extended linearization of the state variables}

\author{
Oscar D. Montoya-Giraldo ${ }^{1}$, Luis F. Grisales-Noreña ${ }^{2}$, \\ Víctor D. Correa-Ramírez ${ }^{3}$ y Didier Giraldo-Buitrago 4
}

Recibido: 19 de septiembre de 2013, Aceptado: 11 de diciembre de 2013

Como citar / How to cite

O. D. Montoya-Giraldo, L. F. Grisales-Noreña, V. D. Correa-Ramírez y D. Giraldo-Buitrago, "Control global del péndulo con rueda de reacción mediante regulación de energía y linealización extendida de las variables de estado", Tecno Lógicas, vol. 17, no. 32, pp. 33-46, 2014.

1 Ingeniero Electricista, Facultad de Ingenierías Eléctrica, Electrónica, Física y de Sistemas y Computación, Universidad Tecnológica de Pereira, Pereira-Colombia, odmontoya@utp.edu.co

2 Ingeniero Electricista, Facultad de Ingenierías Eléctrica, Electrónica, Física y de Sistemas y Computación, Universidad Tecnológica de Pereira, Pereira-Colombia, lufegrisales@utp.edu.co

3 Ingeniero Electricista, Facultad de Ingenierías Eléctrica, Electrónica, Física y de Sistemas y Computación, Universidad Tecnológica de Pereira, Pereira-Colombia, victordc@utp.edu.co

4 M.Sc. en Ingeniería, Facultad de Ingenierías Eléctrica, Electrónica, Física y de Sistemas y Computación, Universidad Tecnológica de Pereira, Pereira-Colombia, dgiraldo@utp.edu.co 


\section{Resumen}

En este documento se presenta el diseño y la simulación de un controlador global para el péndulo invertido con rueda de reacción empleando métodos de regulación de energía y linealización extendida de las variables de estado. La regulación de energía propuesta se fundamenta en la variación gradual de la energía del sistema para alcanzar la posición vertical invertida. La señal de entrada requerida se obtiene a partir de la aplicación del teorema de estabilidad de Lyapunov. El control por realimentación extendida de las variables de estado se emplea para obtener una función no lineal suave que extienda la región de operación del sistema en un rango mayor, en contraste con la realimentación estática de las variables de estado que se obtiene mediante métodos de linealización aproximada alrededor de un punto de operación. El controlador diseñado opera a partir de la conmutación de las señales de control dependiendo de la región de operación y para verificar su robustez y eficiencia se aplican perturbaciones en la señal de control y en las variables medidas. Finalmente las simulaciones y pruebas realizadas sobre el modelo del sistema físico, permiten observar la versatilidad y funcionalidad del controlador propuesto en toda la región de operación del péndulo.

\section{Palabras clave}

Control global por conmutación, linealización extendida, péndulo con rueda de reacción, regulación de energía, variables de estado.

\section{Abstract}

This paper presents the design and simulation of a global controller for the Reaction Wheel Pendulum system using energy regulation and extended linearization methods for the state feedback. The proposed energy regulation is based on the gradual reduction of the energy of the system to reach the unstable equilibrium point. The signal input for this task is obtained from the Lyapunov stability theory. The extended state feedback controller design is used to get a smooth nonlinear function that extends the region of operation to a bigger range, in contrast with the static linear state feedback obtained through the method of approximate linearization around an operating point. The general designed controller operates with a switching between the two control signals depending upon the region of operation; perturbations are applied in the control signal and the (simulated) measured variables to verify the robustness and efficiency of the controller. Finally, simulations and tests using the model of the reaction wheel pendulum system, allow to observe the versatility and functionality of the proposed controller in the entire operation region of the pendulum.

\section{Keywords}

Switching global control, extended linearization, reaction wheel pendulum, energy regulation, state variables. 
O. D. Montoya-Giraldo et al. / Control global del péndulo con rueda de reacción mediante regulación de energía y linealización extendida de las variables de estado

\section{INTRODUCCIÓN}

El péndulo con rueda de reacción hace parte de la familia de péndulos invertidos con una dinámica no lineal compleja, que lo hace altamente atractivo para ser analizado bajo diferentes esquemas de control, tanto locales como globales.

Los sistemas pendulares presuponen un gran desafío para las técnicas clásicas de control, por lo cual desde hace varias décadas se han empleado diferentes metodologías para el diseño de controladores, entre las que se destacan las técnicas de optimización, tales como los algoritmos genéticos y recocido simulado [1], [2], así como las redes neuronales [3], [4], la lógica difusa [5] y las técnicas clásicas de realimentación de variables de estado mediante linealización aproximada [6], controladores PID [7], reguladores óptimos con criterio cuadrático [8] y planos deslizantes [9]; que han sido implementados con gran éxito.

Las características operativas de los péndulos invertidos, han hecho de estos sistemas un banco de pruebas para la compresión de fenómenos físicos asociados a modelos de alta complejidad matemática; razón por la cual, estudios relacionados con la aviación, las telecomunicaciones, el transporte y en general con sistemas de modelado complejo (puentes grúa y lanzamiento de proyectiles) han basado sus sistemas de control en las similitudes encontradas en la operación de dichos sistemas [10]. Por tal motivo en este trabajo se escoge el péndulo invertido con rueda de reacción y se aplican las técnicas de control por regulación de energía y linealización extendida de las variables de estado, con base en el modelo del sistema.

El péndulo invertido con rueda de reacción fue introducido por Mark W. Spong en 1995 con el fin de contribuir con la gama de sistemas no lineales de la familia de los péndulos y proveer un nuevo sistema de prueba para realizar diseños y aplicaciones de gran variedad de técnicas de control [10]. Desde entonces se han desarrollado diversos trabajos que implementan técnicas de control no lineal sobre esta planta; a continuación se describen algunos de los más destacados y cuyo enfoque se ajusta al presente trabajo (a criterio de los autores), sin desconocer que pueden existir otros documentos y desarrollos relevantes.

En 2001 se presenta el péndulo invertido con rueda de reacción como un sistema no lineal apto para la aplicación de diferentes esquemas de control; se desarrollan controladores por realimentación exacta de las variables de estado al igual que linealización aproximada y se emplean funciones de energía para realizar el balanceo del sistema. Los controladores son implementados en un sistema real [10].

Los autores de [11] presentan en 2004 el desarrollo de controladores mediante tres métodos de linealización para la región de operación, se emplean la linealización aproximada de las variables de estado, la disyunción del modelo no lineal del sistema para obtener dos modelos separados con dinámicas lineales y no lineales. Un tercer modelo se desarrolla con base en la reducción de orden del modelo inicial del sistema y por último, para lograr la ubicación del sistema alrededor de la región de operación, se utiliza un controlador difuso. La validación de resultados se hace en tiempo en real para un prototipo del sistema péndulo invertido con rueda de reacción.

En 2007 se presenta un controlador basado en regulación de energía para el balanceo del sistema y un controlador basado en linealización aproximada del modelo del sistema para obtener las ganancias de realimentación de los estados estimados por un observador. Se utiliza un conmutador para seleccionar la señal de control dependiendo de la región de operación. Los resultados se validan en un sistema real [12]. Este mismo año se desarrolla un controlador conmutado para el sistema considerando incertidumbre paramétrica, se realiza un control adaptativo para estimar los parámetros del sistema y un controla- 
dor que varía la posición del sistema a partir de mediciones con el fin de acercarlo a la región de operación. Se implementa el controlador en prototipo real del sistema conocido como Mechkit [13].

En 2011 se desarrolla un modelo para la estabilización y control de un péndulo rotacional invertido basando en la velocidad con la que varía la energía del sistema. El método clásico de balance por funciones de energía es modificado para asegurar que el sistema alcance la posición de equilibrio inestable, desde cualquier condición inicial. Una vez el sistema está en las proximidades de la región de operación es empleado un método de conmutación que intercambia las señales de control, del balance por modelos energéticos a un controlador basado en planos deslizantes. Los resultados numéricos obtenidos al evaluar el modelo a través de métodos de simulación, reflejan la robustez y eficiencia de la metodología propuesta [14].

Como aporte fundamental en este proyecto, se destaca la combinación de dos modelos no lineales para representar el comportamiento global del sistema y la integración de los controladores a través de la definición de tres zonas operativas para la conmutación de la señal de control, con lo que es suavizado el esfuerzo sobre el actuador y la saturación en los sensores. Adicional a esto, es presentado en detalle la formulación por realimentación extendida de las variables de estado para un sistema no lineal, destacando las ventajas de una formulación robusta y poco empleada en la literatura especializada y la industria.

Este artículo es presentado de la siguiente manera. La sección 2 corresponde a la determinación del modelo matemático del sistema físico y la formulación por funciones de energía para el diseño del controlador de balance. En la sección 3 se presenta la formulación clásica de controladores por linealización aproximada, se muestra el desarrollo del controlador por linealización extendida y presenta el modelo que integra los dos controladores. En el apartado 4 se presenta las pruebas desde simulación para el sistema péndulo con rueda de reacción y los diferentes efectos que causan las perturbaciones externas en su operación. En la parte final del documento se presentan las conclusiones y trabajos futuros.

\section{MODELO DEL SISTEMA FíSICO $Y$ DETERMINACIÓN DE LA SEÑAL DE CONTROL POR REGULACIÓN DE ENERGÍA}

\subsection{Descripción y modelado de la planta}

El péndulo con rueda de reacción consta de una barra que tiene una rueda giratoria acoplada a un motor DC en su extremo libre. El par aplicado por el motor se usa activamente para controlar el sistema, el cual está provisto de sensores de movimiento que entregan la posición relativa del ángulo para la rueda de reacción y la posición angular de la barra. La Fig. 1 presenta una representación bidimensional del péndulo y las variables de interés. Los ángulos $\phi$ y a se obtienen de los sensores. El ángulo del péndulo es $\phi$ y la medida absoluta del ángulo $\theta$ de la rueda resulta ser tal como se presenta en (1).

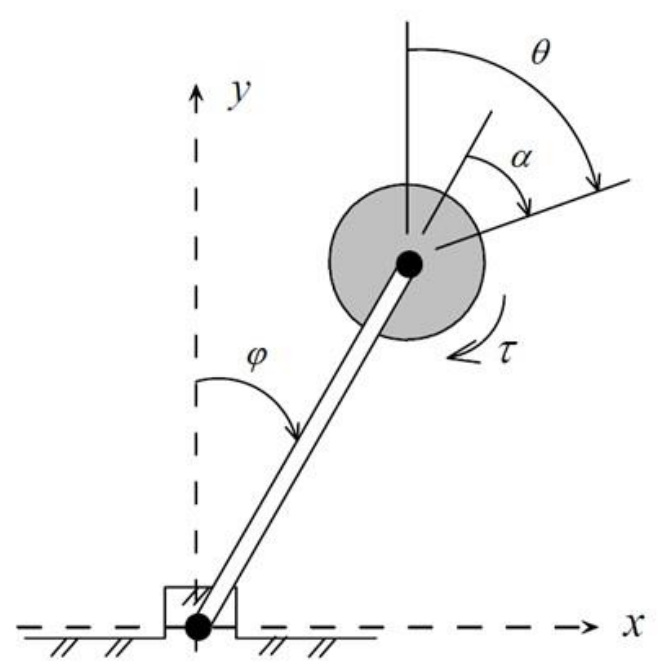

Fig. 1. Modelo bidimensional del péndulo con rueda de reacción. Fuente: Autores 
$\theta=\phi+\alpha$

El modelo de este sistema ha sido tratado y desarrollado ampliamente en diferentes publicaciones como por ejemplo [5], [10], [11], [15]. En la mayoría de estos documentos se destaca el uso de la mecánica de Lagrange, de la cual se obtienen las ecuaciones de movimiento (2) y (3).

$J_{p} \ddot{\phi}=m g l \sin \phi-K_{m} u$

$J_{r} \ddot{\theta}=K_{m} u$

En estas expresiones, $J_{p}$ es el momento de inercia del péndulo con respecto al pivote, $J_{r}$ es el momento de inercia de la rueda con respecto a su centro, $m$ representa la masa del péndulo y $l$ su longitud (desde el pivote hasta el centro de gravedad). La constante $K_{m}$ está relacionada con parámetros físicos del motor y con el factor de escala necesario para expresar la entrada de control como un valor normalizado en el rango $-10 \leq u \leq 10$ unidades de ingeniería (UI). Una presentación simplificada del modelo dado en (2) y (3) puede ser definida como (4) y (5).

$\ddot{\phi}=a \sin \phi-b u$
$\ddot{\theta}=c u$

Siendo $a, b$ y $c$ constantes que dependen de los parámetros del sistema. En este documento se toman los valores asociados al prototipo de prueba (Mechatronics control Kit) presentado en [15], los cuales son: $a=78,4 ; b=1,08$ у $c=198$.

En este modelo dinámico se ha despreciado la fricción en ambos ejes (péndulo y rueda). Dado que la rueda de inercia es simétrica con respecto a su eje de giro, el valor del ángulo $\theta$ es irrelevante y se puede plantear un modelo de estados de orden reducido tomando como variables el ángulo del péndulo, la velocidad del péndulo y la velocidad de la rueda, es decir, $x_{1}=\phi$, $x_{2}=\dot{\phi}, \quad x_{3}=\dot{\theta}$. Al tomar las derivadas temporales de cada una de estas variables y usando (4) y (5), se obtiene el modelo de estados no lineal que se presenta en (6).

$$
\begin{aligned}
& \dot{x}_{1}=x_{2} \\
& \dot{x}_{2}=a \sin x_{1}-b u \\
& \dot{x}_{3}=c u
\end{aligned}
$$

Una representación vectorial de este modelo tiene la forma $\dot{x}=f(x, u)$, donde $x$ es el vector de estados y $f: R^{4} \rightarrow R^{3}$ es la función vectorial no lineal asociada al sistema, en forma matricial el vector de estados y la función no lineal se pueden formular como (7).

$$
x=\left[\begin{array}{l}
x_{1} \\
x_{2} \\
x_{3}
\end{array}\right] \quad f(x, u)=\left[\begin{array}{c}
x_{2} \\
a \sin x_{1}-b u \\
c u
\end{array}\right]
$$

El péndulo con rueda de reacción es un sistema sub-actuado (tiene dos grados de libertad y una entrada de control) y por tanto las estrategias de control que se le aplican tienen la finalidad de alcanzar los puntos de equilibrio y mantener los estados del sistema en esos puntos [5]. En este sistema en particular, los puntos de equilibrio se obtienen al igualar a cero la función $f(x, u)$ considerando entrada cero. De este análisis se obtienen dos puntos de equilibrio: Equilibrio inestable: $\theta=0 ; \phi=0,2 \pi$, $4 \pi, \ldots$ (posición invertida); y equilibrio estable: $\theta=0 ; \phi= \pm \pi, 3 \pi, 5 \pi, \ldots$ (posición de equilibrio natural). El objetivo fundamental de este trabajo es llevar el péndulo a su posición invertida y mantenerlo allí.

\subsection{Regulación de la energía del sistema}

El controlador basado en la linealización extendida del modelo, actuará alrededor de la posición de equilibrio invertida. Para llevar el péndulo de su posición de equilibrio natural al estado invertido se requiere una estrategia de control adicional con el fin de evitar hacerlo manual- 
mente. Entre las opciones más usadas se seleccionó la regulación de energía por la sencillez en su implementación [3], [16]. La estrategia consiste en plantear una función de energía que sea cero en el punto de equilibrio alrededor del cual se desea controlar el sistema. Luego se define una entrada de control con la cual dicha energía sea llevada a cero de forma gradual. En el sistema de la Fig. 1, la relación entre el ángulo del péndulo y su energía total se establece como (8).

$$
E_{p}=\frac{1}{2} J_{p}(\dot{\phi})^{2}+m g l(\cos \phi-1)
$$

La energía cinética de la rueda $\left(\frac{1}{2} J_{p}(\dot{\theta})^{2}\right)$ no se incluye en esta expresión debido a que la velocidad $\dot{\theta}$ no afecta el valor del ángulo $\phi$. Al calcular la derivada temporal de la energía del péndulo se obtiene la expresión (9).

$\dot{E}_{p}=\dot{\phi}\left(J_{p} \ddot{\phi}-m g l \sin \phi\right)$

Que al ser comparada con (2) en el modelo del sistema resulta ser (10).

$\dot{E}_{p}=-\dot{\phi} K_{m} u$

$\mathrm{Al}$ analizar la función de energía dada por (8), se observa que toma su valor mínimo (negativo) cuando el péndulo se encuentra en su posición de equilibrio natural (abajo) y se hace cero en la posición invertida. La señal de control llevará esta energía a cero si se garantiza que $\dot{E} p>0$. De (10) se observa que si el producto $\dot{\phi} u$ es negativo se logra la condición deseada. Existen diferentes opciones, entre las cuales se destaca la expresión (11), que usa la energía y el signo de la velocidad del péndulo.

$u=K_{u} E_{p} \operatorname{sgn}(\dot{\phi})$
En esta expresión, sgn $(x)$ es la función signo. La ganancia $K_{u}$ permite manipular la amplitud de la señal reguladora y la rapidez con la que el péndulo alcanza la posición invertida; su valor se ajusta de forma empírica. El término sgn $(\dot{\phi})$ permite que la señal de control refuerce el movimiento del péndulo (se aplica torque en la misma dirección de movimiento), con lo que se logra una oscilación de amplitud creciente hasta alcanzar la posición invertida.

\section{DISEÑO DEL CONTROLADOR POR REALIMENTACIÓN DE LAS VARIABLES DE ESTADO}

Uno de los métodos clásicos más conocido en la literatura para el control de sistemas físicos es la realimentación del vector de estados. Este método se basa en la representación del modelo dinámico del sistema empleando un conjunto de ecuaciones diferenciales de primer orden (modelo en variables de estado). El vector de estado contiene el conjunto de variables que poseen la información necesaria para desarrollar una estrategia de control por realimentación; sin embargo es necesario verificar las condiciones de controlabilidad (y observabilidad) antes de plantear la estrategia de control [17].

\subsection{Método de linealización aproximada}

Una representación general del modelo de estados de un sistema no lineal de orden $n$ tiene asociadas (12) y (13).

$$
\begin{aligned}
& \dot{x}=f(x, u) \\
& y=g(x)
\end{aligned}
$$

En el caso de una entrada y una salida (Single Input - Single Output o SISO), $x$ es un vector de $R^{n}, f(x, u)$ es una función vectorial suave $\left(f: R^{n+1} \rightarrow R^{n}\right)$ y $g(x)$ es una 
función escalar suave $\left(\mathrm{g}: R^{n} \rightarrow R\right)$. Para usar estrategias de control lineal alrededor de un punto de operación $x_{0}$ dado, se obtiene un modelo linealizado considerando los términos de primer orden de la expansión en serie de Taylor, es decir, las expresiones (14) y (15).

$$
\begin{aligned}
& \Delta \dot{x}=\left.\frac{\delta f}{\delta x}\right|_{x_{0}} * \Delta x+\left.\frac{\delta f}{\delta u}\right|_{x_{0}} * \Delta u \\
& \Delta y=\left.\frac{\delta g}{\delta x}\right|_{x_{0}} * \Delta x
\end{aligned}
$$

$\mathrm{Al}$ evaluar las derivadas en el punto de operación se obtienen las matrices del modelo linealizado: $\quad A=\delta f /\left.\delta x\right|_{x_{0}}$; $B=\delta f /\left.\delta u\right|_{x_{0}} ; C=\delta g /\left.\delta x\right|_{x_{0}}$ Alrededor del punto de operación se obtiene entonces el modelo de estados lineal dado por (16).

$$
\begin{aligned}
& \Delta \dot{x}=A \Delta x+B \Delta u \\
& \Delta y=C \Delta x
\end{aligned}
$$

En el cual $\Delta x, \Delta u$ y $\Delta y$ representan las variaciones de los estados, la entrada y la salida respectivamente alrededor del punto de operación definido $\left(\Delta x=x-x_{0}\right.$, $\left.\Delta u=u-u_{0}, \Delta y=y-y_{0}\right)$.

Sobre el modelo linealizado se puede diseñar un regulador lineal de la forma $\Delta u=-K \Delta x$ (siempre que el par $(A, B)$ sea controlable). Las ganancias de realimentación $K=\left[\begin{array}{llll}K_{1} & K_{2} & \ldots & K_{n}\end{array}\right]$ se calculan de forma que en lazo cerrado se obtengan los polos o valores propios deseados [17].

\subsection{Método de linealización extendida}

Si en el modelo linealizado del sistema no se especifica un punto de operación dado, se obtienen expresiones genéricas en función de los estados del punto de operación $x_{0}=\left[\begin{array}{llll}x_{10} & x_{20} & \ldots & x_{n 0}\end{array}\right]^{\mathrm{T}}$. Al diseñar un regulador, las ganancias $\bar{K}$ quedan también definidas en términos de $x_{0}$, lo que permite proponer una ley de control no lineal de la forma (17).

$u=-K(x)$

Que sea una extensión del regulador lineal alrededor de $x_{0}$ y cuya linealización corresponde a la expresión (18).

$u=-\left.\frac{\delta K}{\delta x_{1}}\right|_{x_{0}} * \Delta x_{1}-\left.\frac{\delta K}{\delta x_{2}}\right|_{x_{0}} * \Delta x_{2}-\cdots-\left.\frac{\delta K}{\delta x_{n}}\right|_{x_{0}} * \Delta x_{n}$

Si $\bar{K}=\left[\begin{array}{llll}\overline{K_{1}} & \overline{K_{2}} & \ldots & \overline{K_{n}}\end{array}\right]$ es el regulador lineal clásico alrededor del punto de operación $(\Delta u=-K \Delta x)$, se impone el conjunto de condiciones (19) sobre la función no lineal $K(x)$ con el fin de que ambos controladores coincidan en el punto $x_{0}$.

$\left.\frac{\delta K}{\delta x_{i}}\right|_{x_{0}}=\bar{K}_{i} ; i=1,2, \ldots, n$

Si no se ha especificado un punto de operación numérico, es posible hallar una expresión para $K(x)$ integrando estas condiciones con respecto a las variables $\begin{array}{llll}x_{10} & x_{20} & \ldots & x_{n 0} . \text { Aunque este problema pue- }\end{array}$ de tener múltiples soluciones, se puede obtener un resultado sencillo si $K(x)$ se define de una forma conveniente [18].

\subsection{Linealización extendida aplicada al péndulo con rueda de reacción}

Por ser un sistema sub-actuado, el péndulo con rueda de reacción solo se puede controlar en las posiciones de equilibrio (la posición natural $\phi= \pm \pi, \pm 3 \pi, \pm 5 \pi, \ldots$ o la invertida $\phi=0, \pm 2 \pi, \pm 4 \pi, \ldots)$. Aunque inicialmente se considera un punto de operación arbitrario en el cálculo del regulador no lineal $u=-K(x)$, posteriormente se escogerá la posición invertida para realizar las simulaciones.

$\mathrm{Si}$ el conjunto de (6) que describe la dinámica del modelo se linealiza alrededor 
de un punto de operación $x_{0}$ usando (14) y (15) se obtienen (20) y (21)

$$
\begin{aligned}
& {\left[\begin{array}{l}
\Delta \dot{x}_{1} \\
\Delta \dot{x}_{2} \\
\Delta \dot{x}_{3}
\end{array}\right]=\left[\begin{array}{ccc}
0 & 1 & 0 \\
a \cos x_{1} & 0 & 0 \\
0 & 0 & 0
\end{array}\right]\left[\begin{array}{l}
\Delta x_{1} \\
\Delta x_{2} \\
\Delta x_{3}
\end{array}\right]+\left[\begin{array}{c}
0 \\
-b \\
c
\end{array}\right] \Delta u} \\
& \Delta y=\left[\begin{array}{lll}
1 & 0 & 0
\end{array}\right]\left[\begin{array}{l}
\Delta x_{1} \\
\Delta x_{2} \\
\Delta x_{3}
\end{array}\right]
\end{aligned}
$$

Donde se pueden identificar las matrices $A, B$ y $C$ mostradas en (16). La matriz de controlabilidad asociada a este modelo se define como (22).

$$
W_{c}=\left[\begin{array}{lll}
A & A B & A^{2} B
\end{array}\right]=\left[\begin{array}{ccc}
0 & -b & 0 \\
-b & 0 & -a b \cos x_{1} \\
c & 0 & 0
\end{array}\right]
$$

$$
\mathrm{Y} \quad \text { cuyo determinante es }
$$
$\operatorname{det}\left(W_{c}\right)=a b^{2} c \cos x_{10}$, lo que implica que el sistema es controlable ( $W_{c}$ tiene rango tres), excepto en los puntos de operación $\phi= \pm \pi / 2$ o equivalentes. Se plantea una señal de control como se indica en (17) $u=-K(x)$ con la definición presentada en (23) y se impone la condición (24).

$$
\begin{aligned}
& u=-K_{1}(x)-K_{2}(x)-K_{3}(x) \\
& \left.\frac{\delta K}{\delta x_{i}}\right|_{x_{0}}=\bar{K}_{i} ; i=1,2,3
\end{aligned}
$$

Siendo $\bar{K}=\left[\begin{array}{lll}\overline{K_{1}} & \overline{K_{2}} & \overline{K_{3}}\end{array}\right]$ la matriz de ganancias del regulador lineal en la posición invertida $\phi=0$ (y sus equivalentes). Este último se obtiene asignando valores propios adecuados a la matriz de lazo cerrado $A_{L C}=A-B \bar{K}$, es decir, como (25).

$$
|s I-(A-B \bar{K})|=\left(s+\lambda_{1}\right)\left(s+\lambda_{2}\right)\left(s+\lambda_{3}\right)
$$

$\mathrm{Al}$ realizar las operaciones indicadas en (24) usando las matrices de (20) y (21) y el vector $\bar{K}$ se obtienen los resultados (26), (27) y (28).

$$
\begin{aligned}
& \bar{K}_{1}(x)=-\frac{\beta+a \cos x_{10}}{b}=\frac{\delta K_{1}}{\delta x_{10}} \\
& \bar{K}_{2}(x)=-\frac{1}{b}\left(\frac{\gamma}{a} \sec x_{10}+\alpha\right)=\frac{\delta K_{2}}{\delta x_{20}} \\
& \bar{K}_{3}(x)=-\frac{\gamma}{a c} \sec x_{10}=\frac{\delta K_{3}}{\delta x_{30}}
\end{aligned}
$$

Las constantes $\alpha, \beta$ y $\gamma$ se relacionan con los valores propios deseados $\left(\lambda_{1}, \lambda_{2}, \lambda_{3}\right)$ mediante las expresiones $\alpha=\lambda_{1}+\lambda_{2}+\lambda_{3}$, $\beta=\lambda_{1} \lambda_{2}+\lambda_{1} \lambda_{3}+\lambda_{2} \lambda_{3}, y \gamma=\lambda_{1} \lambda_{2} \lambda_{3}$.

De las ecuaciones (26), (27) y (28) se obtienen las funciones $K_{1}(x), K_{2}(x)$ y $K_{3}(x)$, si se integra en cada una con respecto a variables que sustituyen a los valores de punto de operación, respectivamente $x_{10}$, $x_{20} y x_{30}$. Los resultados se muestran en (29), (30) у (31).

$K_{1}(x)=-\int_{x_{10}}^{x_{1}} \frac{\beta+a \cos v}{b} d v=\frac{\beta\left(x_{10}-x_{1}\right)+a\left(\sin x_{10}-\sin x_{1}\right)}{b}$

$K_{2}(x)=-\int_{x_{20}}^{x_{2}} \frac{1}{b}\left(\frac{\gamma}{a} \sec x_{10}+\alpha\right) d v=\frac{1}{b}\left(\frac{\gamma}{a} \sec x_{1}+\alpha\right)\left(x_{20}-x_{2}\right)(30)$

$\bar{K}_{3}(x)=-\int_{x_{30}}^{x_{3}} \frac{\gamma}{a c} \sec x_{10} d v=\frac{\gamma}{a c}\left(x_{30}-x_{3}\right) \sec x_{1}$

Al remplazar (29), (30) y (31) en (22) se obtiene el regulador no lineal que extiende a su equivalente linealizado $(\Delta u=-K \Delta x)$, alrededor del punto de operación $x_{10}=0$ $(\phi=0)$.

\subsection{Integración de los controladores}

En la sección denominada regulación de la energía del sistema se presentó un controlador que se encarga de llevar el péndulo desde la posición de reposo hasta una zona cercana a la posición invertida [16]. En esta zona se aplica la acción del control generado a partir de la linealización extendida del modelo (linealización extendida aplicada al péndulo con rueda de reacción). Para evitar que el péndulo llegue con una velocidad elevada a la posición invertida y que se presente una transición brusca entre los controladores, las regiones de acción de estos se definen cuidadosamente dejando una franja en la cual ninguno de 
O. D. Montoya-Giraldo et al. / Control global del péndulo con rueda de reacción mediante regulación de energía y linealización extendida de las variables de estado

los dos actúa. En la Fig. 2 se presentan las franjas de acción de ambos controladores. El regulador de energía opera en un rango alrededor de la posición de reposo (zona 1) y cuya amplitud puede ajustarse para modificar el tiempo de subida. Por su parte, el controlador por linealización extendida actúa alrededor del punto de equilibrio inestable (zona 2).

El diagrama de bloques presentado en la Fig. 3, corresponde a la propuesta de implementación del controlador global para el péndulo con rueda de reacción.

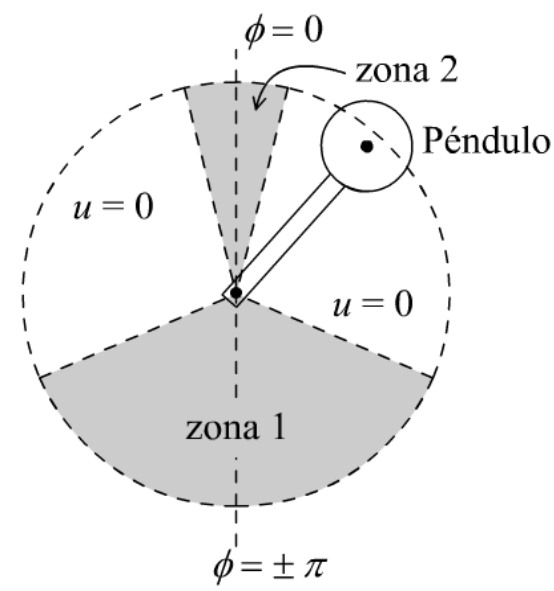

Fig. 2. Zonas de actuación de los controladores. Fuente: Autores

\section{PRUEBAS Y RESULTADOS}

Para la implementación del controlador global para el péndulo con rueda de reacción, se empleó el software de programación y simulación MATLAB 2010a en un computador Intel Celeron, con sistema operativo Windows 7 profesional y $2 \mathrm{~GB}$ RAM.

Para el péndulo con rueda de reacción se realizan tres pruebas. La primera asociada a la correcta operación del esquema de control para regulación y dos pruebas adicionales que corresponden a la inclusión de señales erróneas o perturbaciones en el sensor de posición angular y en la señal de control, las cuales son generadas por métodos de simulación [3].

Con el fin de visualizar adecuadamente el comportamiento dinámico del sistema en relación con las velocidades angulares del péndulo y la rueda de reacción, se realizó una normalización en función de sus valores máximos, es decir, que sus gráficas son mostradas como un porcentaje de los valores extremos, es decir, para la velocidad angular del péndulo $17,2 \mathrm{rad} / \mathrm{s}$ y para la rueda de reacción $900 \mathrm{rad} / \mathrm{s}$.

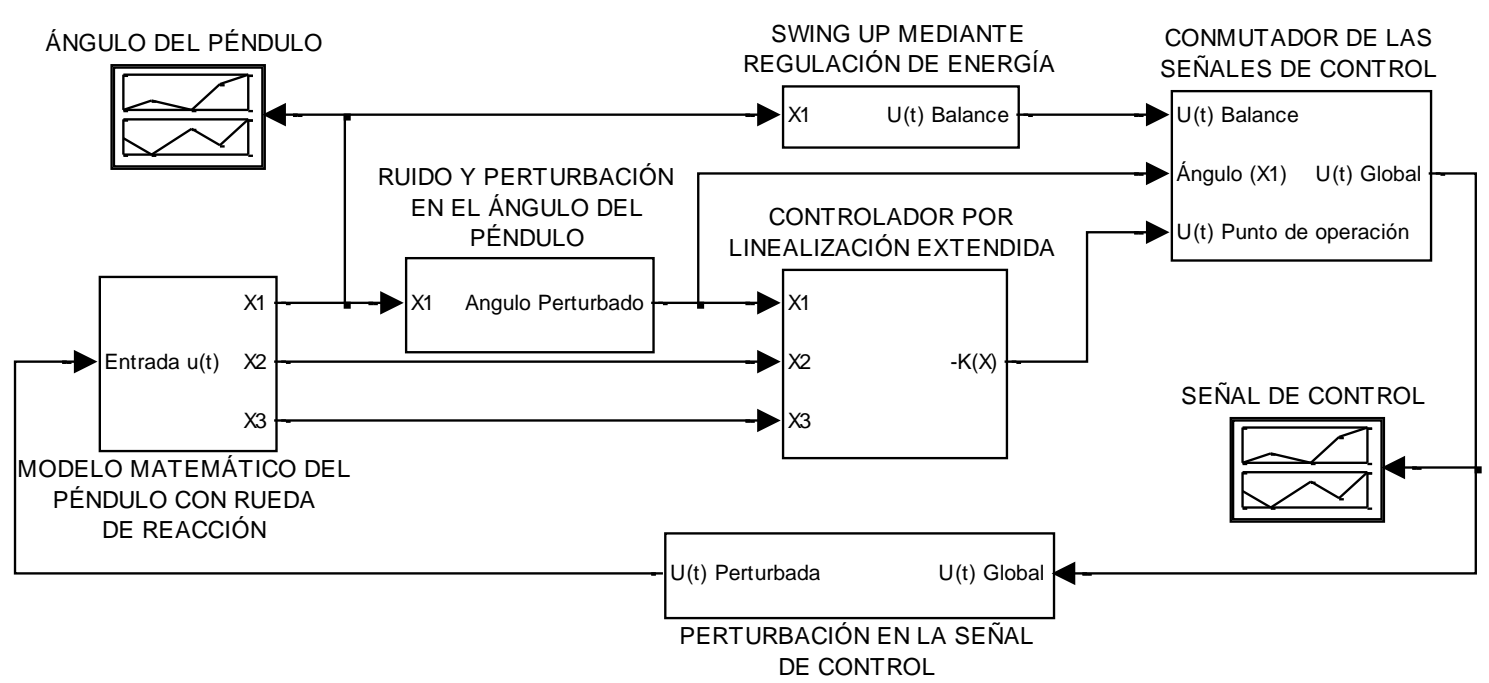

Fig. 3. Diagrama de bloques para el controlador global del péndulo con rueda de reacción usando realimentación extendida y regulación de energía. Fuente: Autores 
O. D. Montoya-Giraldo et al. / Control global del péndulo con rueda de reacción mediante regulación de energía y linealización extendida de las variables de estado

\subsection{Estabilización del sistema alrededor del punto de operación}

Esta prueba es conocida como regulación de las variables de estado y se constituye como una prueba obligatoria para mostrar que el esquema de control propuesto es adecuado ante el caso base. La Fig. 4 muestra el comportamiento del péndulo ante la aplicación de las señales de control, cuando su posición inicial es la posición de reposo estable, es decir, la posición vertical inferior.

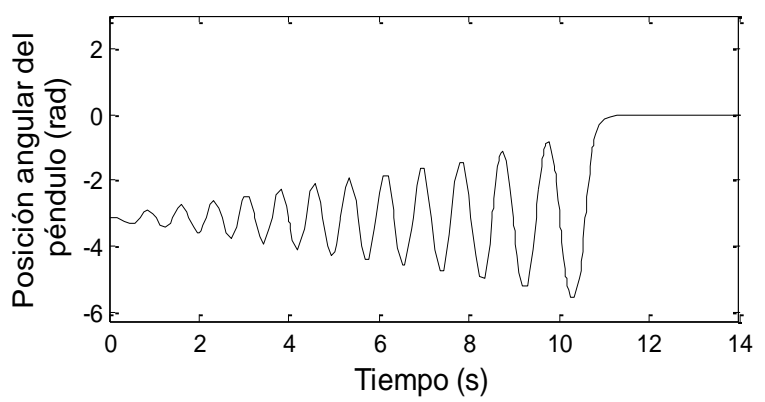

Fig. 4. Posición angular del péndulo en función del tiempo. Fuente. Autores

Como se observa en la Fig. 4, el tiempo que tarde el péndulo en alcanzar el punto de operación en la posición vertical superior es de $11 \mathrm{~s}$ aproximadamente, tiempo durante el cual presenta oscilaciones de amplitud creciente, hasta alcanzar el punto de entrada en operación del controlador local. Por otro lado, la Fig. 5 muestra como la energía de la planta varía desde el punto de mínima energía (posición de reposo natural) hasta el valor máximo (punto de operación deseado), y que según el modelo desarrollado toma una magnitud de cero, según se presentó en (8).

Las velocidades angulares del péndulo se muestran en la Fig. 6, de donde es posible inferir que son equivalentes al 100\% de su magnitud real por unos pocos segundos, evento que se presenta cuando la amplitud del balance aumenta a valores muy próximos a la región de operación en la posición vertical superior.

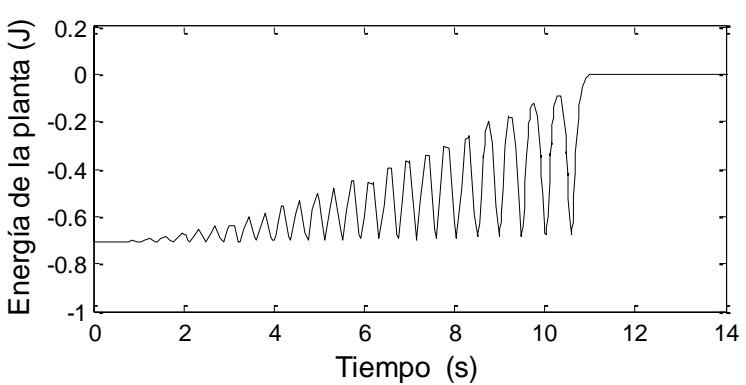

Fig. 5. Variación de la energía en función del tiempo. Fuente. Autores

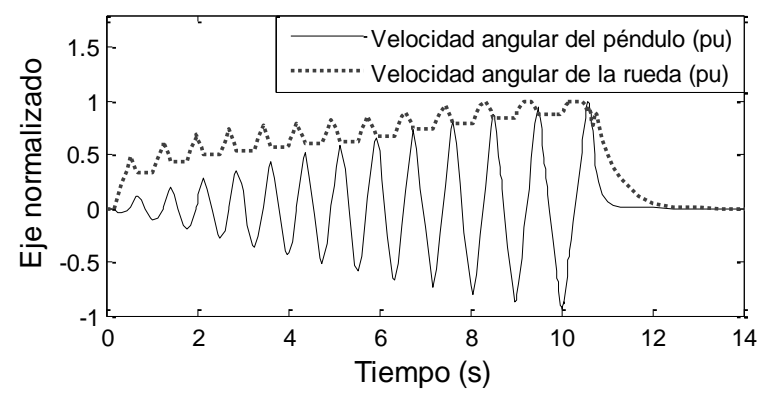

Fig. 6. Comportamiento porcentual de las velocidades. Fuente. Autores

La señal de control global que hace posible el balanceo del sistema y la estabilización del sistema alrededor del punto de operación, se observa en la Fig. 7, y es el resultado de la conmutación de las leyes de control propuesta en el diagrama de bloques de la Fig. 3. Por otro lado de la Fig. 7, es posible inferir que la señal de control obtenida por regulación de energía, funciona como un inversor de corriente, y que se traduce como una polaridad alterna en los terminales del actuador, permitiendo así que el péndulo se balance a medida que su energía tiende a cero.

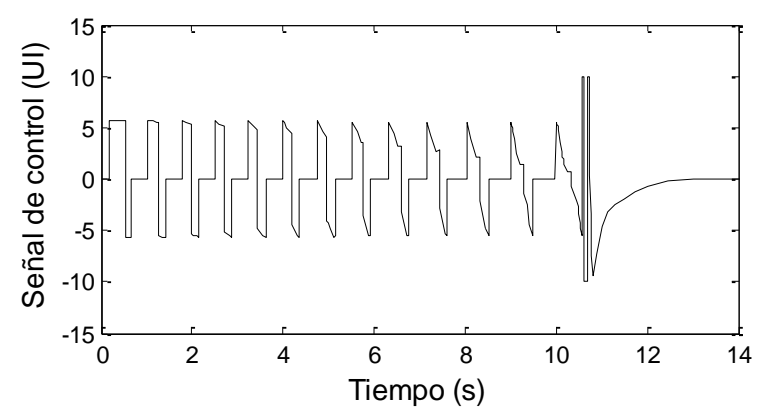

Fig. 7. Señal de control en función del tiempo. Fuente: Autores 
O. D. Montoya-Giraldo et al. / Control global del péndulo con rueda de reacción mediante regulación de energía y linealización extendida de las variables de estado

\subsection{Perturbaciones en el sensor de posición angular del péndulo}

Los sistemas de medición presentes en la industria, debido a las condiciones normales de operación son comúnmente afectados por interferencias electromagnéticas que son generadas en el propio ambiente de trabajo, razón por la cual, cuando se diseña el control, es necesario simular comportamientos atípicos en los sensores y elementos de medida, con el fin de evidenciar la robustez y eficiencia de los controladores ante señales externas y/o perturbaciones indeseadas.

En la Fig. 8 se muestra un ruido típico al que se enfrentan los equipos de medida en ambientes industriales y el cual es inducido sobre el sensor de posición angular del péndulo para emular un comportamiento inusual en las medidas.

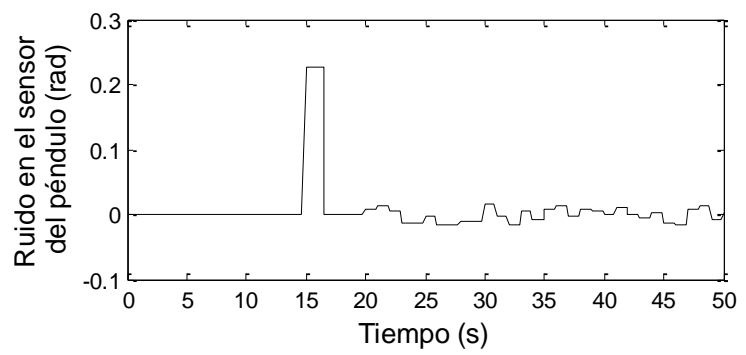

Fig. 8. Señal de ruido inducida en el sensor de posición angular. Fuente: Autores

Por otro lado, la Fig. 9 presenta el comportamiento de la posición angular del péndulo, en presencia de datos erróneos en la señal medida por su sensor; es posible observar que cuando se presenta un pico en la señal medida alrededor de los $15 \mathrm{~s}$ el péndulo pierde estabilidad y sale de la posición de equilibrio, sin embargo el controlador por regulación de energía, tras una única oscilación del péndulo, lo lleva nuevamente a la posición de equilibrio estable. Sólo se presentan pequeñas variaciones en la posición angular inducidas por las medidas tomadas por el sensor después de los $22 \mathrm{~s}$, logrando el controlador mantenerlo alrededor del punto de operación sin mayores esfuerzos.

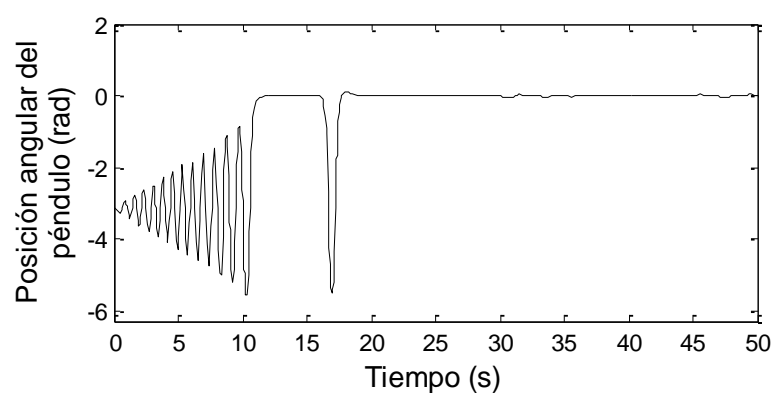

Fig. 9. Posición angular del péndulo ante perturbaciones en su sensor. Fuente: Autores

Las velocidades angulares normalizadas del péndulo y la rueda de reacción se muestran en la Fig. 10 y es posible observar que en el momento en que la posición angular del péndulo sale de la región de operación, la rueda se acelera hasta llegar a la velocidad nominal, con el fin de llevar la planta nuevamente a la zona de operación. Para la señal de ruido presente en el sensor de medida, la velocidad de la rueda tiene cambios que no sobrepasan sus límites y mantienen estable el péndulo en la posición vertical superior, comportamiento que es de esperarse, debido a los errores introducidos en el sistema a causa de la señal errónea presente en el sensor de posición angular.

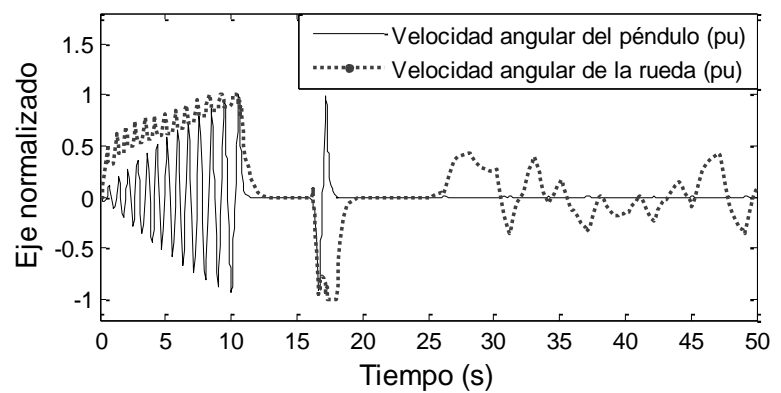

Fig. 10. Velocidades angulares en presencia de ruido. Fuente: Autores

La señal de control que permite estabilizar el sistema desde su posición inicial, hasta el punto de operación en la posición vertical superior se presenta en la Fig. 11. Es posible observar los momentos en que actúan cada una de las leyes de control conmutadas, es decir, cuando el péndulo sale de la región de operación actúa la señal de control por regulación y durante 
las oscilaciones alrededor del punto de operación actúa la señal de control obtenida mediante la linealización extendida de las variables de estado.

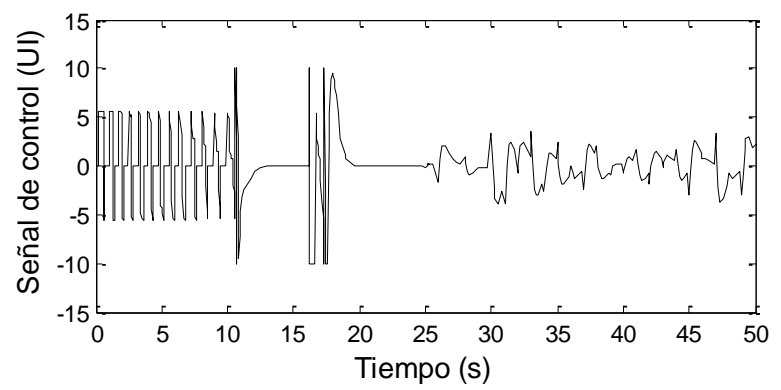

Fig. 11. Señales de control ante perturbaciones. Fuente: Autores

\subsection{Perturbaciones en la señal de control}

Los comportamientos atípicos en los sensores, elementos de medida y actuadores, son comunes cuando los equipos controlados se encuentran sometidos a trabajos pesados en ambientes industriales; razón por la cual es esencial conocer los comportamientos del sistema ante eventos ajenos a su operación normal, con el fin de verificar que no se producirán daños o afectaciones físicas en la planta y en los mecanismos de control empleados.

Dado que los controladores normalmente están desarrollados en función de señales eléctricas, estas son propensas a perturbaciones debidas a fenómenos electromagnéticos, razón por la cual se inducen señales de tipo pulso en la señal de control, que producen comportamientos indeseados sobre la planta. La Fig. 12, muestra una señal de control con alteraciones producidas por pulsos de corta duración.

De la Fig. 12, se observa que existen dos alteraciones de la señal de control, la primera se presenta a los $15 \mathrm{~s}$, cuando un pulso de magnitud 3UI y duración de $0,5 \mathrm{~s}$, y un segundo pulso de duración $0,25 \mathrm{~s}$ y magnitud 8UI, las cuales producen variaciones en la posición angular de la planta, tal como se muestra en la Fig. 13.

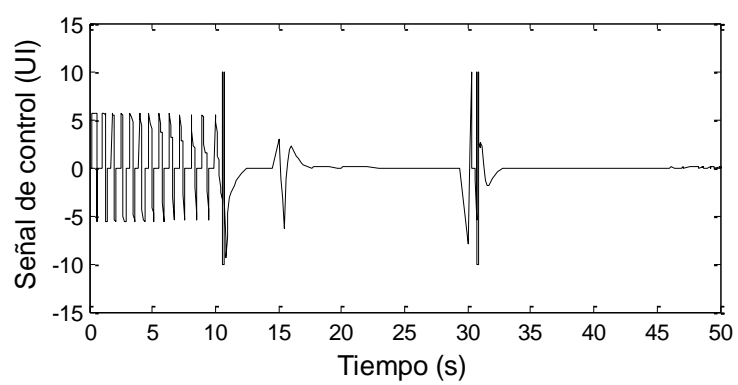

Fig. 12. Señal de control presencia de perturbaciones. Fuente: Autores

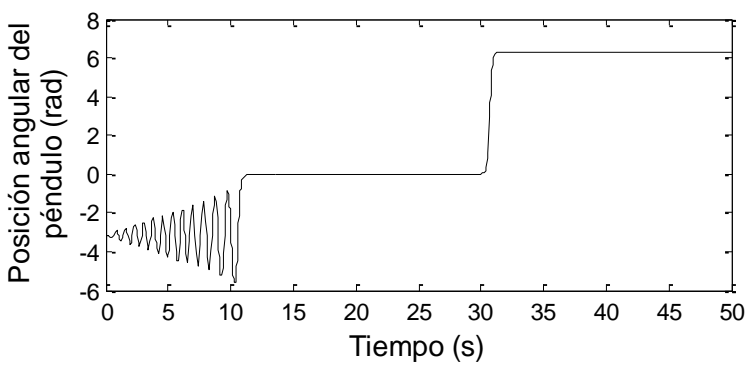

Fig. 13. Posición angular del péndulo ante perturbaciones en la señal de control. Fuente: Autores

En la Fig. 13 es claro que la primera perturbación de baja magnitud no altera la operación en el punto de equilibrio y solo se presenta una pequeña variación alrededor del ángulo de referencia; sin embargo a los $30 \mathrm{~s}$, cuando aparece un pulso en la señal de control de mayor magnitud, el punto de operación del sistema cambia dando una vuelta completa, hasta llegar el siguiente punto de operación, este comportamiento físicamente implica que el péndulo después de caer, llega a la posición de equilibrio estable con el impulso que lleva y la actuación de la ley de control por regulación de energía que lo impulsa lo suficiente logrando alcanzar el siguiente punto de equilibrio, sin la necesidad de hacer un balanceo completo, como ocurrió en el evento simulado en la Fig. 9.

El comportamiento de las velocidades angulares del sistema se muestra en la Fig. 14, donde se observa que para las dos perturbaciones en la señal de control la rueda de reacción se acelera con el fin de mantener la planta en su punto de operación y la velocidad de rotación del péndulo 
alcanza un pico cuando la planta cae a los 30 s. Es de notar, que el actuador no solo presenta operación en estado nominal por un corto lapso de tiempo, y este se da cuando las oscilaciones en la posición angular llegan al máximo justo antes de que entre en operación en controlador por realimentación de las variables de estado.

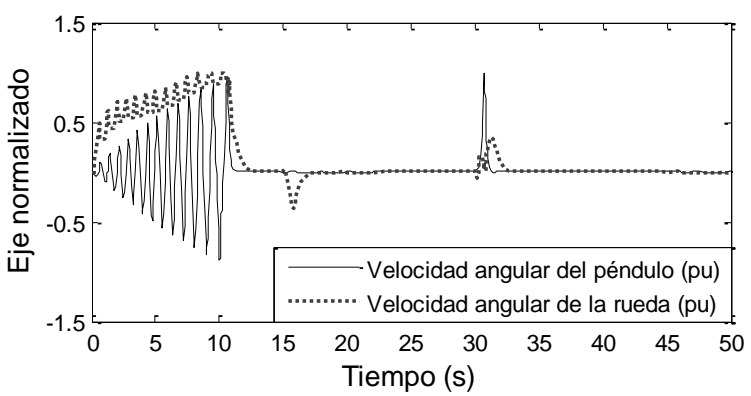

Fig. 14. Variación porcentual de las velocidades angulares del sistema ante perturbaciones en la señal de control. Fuente: Autores

\section{CONCLUSIONES Y TRABAJO FUTURO}

Se desarrolló el diseño de un controlador integrado para el péndulo invertido con rueda de reacción que muestra un comportamiento adecuado en todo el rango de operación de la planta, logrando el objetivo de control y manteniendo la señal de control en una condición libre de saturación, es decir, que no se sobrepasan los límites operativos del actuador.

Se presenta una aplicación de la técnica de control por realimentación de estados mediante linealización extendida, que permite ampliar el rango de operación alrededor del punto de operación a través de las funciones no lineales continuas que sustituyen las ganancias estáticas clásicas, que se obtienen con el método de linealización aproximada.

El control por regulación de energía muestra ser una alternativa sencilla $y$ eficiente para realizar el balanceo de sistemas pendulares. Se logra a través de la manipulación de los estados energéticos de la planta y permite definir señales de control que obedezcan a los criterios del dise- ñador, es decir, que no se comprometa el estado operativo del sistema, o en otras palabras, que ninguna de las señales sobrepase sus límites de operación.

Se propone implementar un control global para el péndulo invertido con rueda de reacción que incluya todos los estados del sistema y en el cual la señal de control sea definida por la aplicación de un voltaje a los terminales del motor y que no desprecie la dinámica presente cuando se modela un motor de corriente continua.

\section{REFERENCIAS}

[1] M. A. Martínez, J. Sanchis, and X. Blasco, "Algoritmos Genéticos Aplicados al Diseño de Controladores Robustos," Rev. Iberoam. automática e informática Ind. (RIAI), vol. 3, no. 1, pp. 39-51, 2006.

[2] J. G. Hoyos, J. E. Cardona, and R. Arango, "Control en linea con algoritmos genéticos y recocido simulado," Sci. Tech., vol. XIII, no. 35, pp. 113-116, 2007.

[3] L. F. Escobar Dávila, O. D. Montoya Giraldo, and D. Giraldo Buitrago, "Control Global del Péndulo de Furuta Empleando Redes Neuronales Artificiales y Realimentación de Variables de Estado," Tecno Lógicas, no. 30, pp. 71-94, 2013.

[4] J. Wu, H. Su, and T. Wu, "ANN Control of Inverted Pendulum," in 2008 First International Conference on Intelligent Networks and Intelligent Systems, 2008, pp. 9-12.

[5] J.-X. Xu, Z.-Q. Guo, and T. H. Lee, "An optimal fuzzy logic controller for an underactuated unicycle," in IECON 2011 - 37th Annual Conference of the IEEE Industrial Electronics Society, 2011, pp. 2335-2340.

[6] Ó. O. Rodríguez Díaz, E. L. Téllez Valderrama, and D. A. Gutiérrez Ramírez, "Simulating a Rotational Inverted Pendulum Model by using Matlab and Easy Java Simulations," Tecno Lógicas, no. 28, pp. 15-32, 2012.

[7] L. B. Prasad, B. Tyagi, and H. O. Gupta, "Modelling and Simulation for Optimal Control of Nonlinear Inverted Pendulum Dynamical System Using PID Controller and LQR," in 2012 Sixth Asia Modelling Symposium, 2012, pp. 138-143.

[8] O. D. Montoya, J. G. Valenzuela, and D. Giraldo, "Control global del Péndulo Rotacional Invertido empleando modelos de energía," Sci. Tech., vol. XVII, no. 52 , pp. 16-25, 2012.

[9] R. Iriarte, L. T. Aguilar, and L. Fridman, "Second order sliding mode tracking controller for inertia wheel pendulum," J. Franklin Inst., vol. 350, no. 1, pp. 92106, Feb. 2013

[10] M. W. Spong, P. Corke, and R. Lozano, "Nonlinear control of the Reaction Wheel Pendulum," Automatica, vol. 37, no. 11, pp. 1845-1851, Nov. 2001.

[11] B. Bapiraju, K. N. Srinivas, P. Kumar P, and L. Behera, "On balancing control strategies for a reaction wheel pendulum," in Proceedings of the IEEE 
O. D. Montoya-Giraldo et al. / Control global del péndulo con rueda de reacción mediante regulación de energía y linealización extendida de las variables de estado

INDICON 2004. First India Annual Conference, 2004., 2004, pp. 199-204.

[12] F. Jepsen, A. Soborg, A. R. Pedersen, and Z. Yang, "Development and control of an inverted pendulum driven by a reaction wheel," in 2009 International Conference on Mechatronics and Automation, 2009, pp. 2829-2834.

[13] A. A. Bobtsov, A. A. Pyrkin, and S. A. Kolyubin, "Adaptive stabilization of a reaction wheel pendulum on moving LEGO platform," in 2009 IEEE International Conference on Control Applications, 2009, pp. 1218-1223.

[14] B. R. Andrievsky, "Global stabilization of the unstable Reaction-Wheel Pendulum," Autom. Remote Control, vol. 72, no. 9, pp. 1981-1993, Sep. 2011.
[15] D. Giraldo, V. D. Correa, and A. Molina, "Control global para el péndulo con rueda de reacción," Sci. Tech., vol. XIII, no. 35, pp. 95-99, 2007.

[16] M. Iwashiro, K. Furuta, and K. J. Astrom, "Energy based control of pendulum," in Proceeding of the 1996 IEEE International Conference on Control Applications, 1996, pp. 715-720.

[17] D. Giraldo and E. Giraldo, Teoría de control análogo. Univ. Tecnológica de Pereira, 2010, p. 287.

[18] H. Sira-Ramírez, R. Márquez, F. Rivas-Echeverría, and O. Llanes-Santiago, Control de Sistemas No Lineales: Linealización aproximada, extendida, exacta. Madrid: Pearson-Prentice-Hall, 2005. 